\title{
Fidelity recovery in chaotic systems and the Debye-Waller factor
}

\author{
H.-J. Stöckmann and R. Schäfer \\ Fachbereich Physik der Philipps-Universität Marburg, D-35032 Marburg, Germany
}

(Dated: July 11, 2018)

\begin{abstract}
Using supersymmetry calculations and random matrix simulations, we studied the decay of the average of the fidelity amplitude $f_{\epsilon}(\tau)=\left\langle\psi(0)\left|\exp \left(2 \pi \imath H_{\epsilon} \tau\right) \exp \left(-2 \pi \imath H_{0} \tau\right)\right| \psi(0)\right\rangle$, where $H_{\epsilon}$ differs from $H_{0}$ by a slight perturbation characterized by the parameter $\epsilon$. For strong perturbations a recovery of $f_{\epsilon}(\tau)$ at the Heisenberg time $\tau=1$ is found. It is most pronounced for the Gaussian symplectic ensemble, and least for the Gaussian orthogonal one. Using Dyson's Brownian motion model for an eigenvalue crystal, the recovery is interpreted in terms of a spectral analogue of the Debye-Waller factor known from solid state physics, describing the decrease of X-ray and neutron diffraction peaks with temperature due to lattice vibrations.

PACS numbers: 05.45.Mt, 05.45.Pq, 03.65.Yz
\end{abstract}

The concept of fidelity has been developed by Peres as a tool to characterize the stability of a quantummechanical system against perturbations 1 ]. It was introduced as the squared modulus of the overlap integral of a wave packet with itself after developing forth and back under the influence of two slightly different Hamiltonians. Very similar concepts had been applied already in the old spin-echo experiments of nuclear magnetic resonance half a century ago (see reference [2] for a review). The renewed interest in the topic results from the idea to realize quantum computers by means of spin systems, where stability against quantum-mechanical perturbations obviously is of vital importance [3] .

Roughly speaking there are three regimes. In the perturbative regime, where the strength of the perturbation is small compared to the mean level spacing, the decay of the fidelity is Gaussian. As soon as the perturbation strength becomes of the order of the mean level spacing, exponential decay starts to dominate, with a decay constant obtained from Fermi's golden rule [4, 5]. For very strong perturbations the decay becomes independent of the strength of the perturbation. Here, in the Loschmidt regime, the decay is still exponential, but now the decay constant is given by the classical Lyapunov exponent [6]. Exactly such a behaviour had been observed experimentally in a spin-echo experiment on isolated spins coupled weakly to a bath of surrounding spins [7].

Gorin et al. [8] calculated the fidelity decay within random matrix theory in the regime of small perturbations and could correctly describe the change from Gaussian to exponential behaviour with increasing perturbation strength. The Lyapunov regime is non-universal and thus not accessible in a random matrix model.

Intuitively, one would expect that in chaotic systems the fidelity decay is stronger than in integrable ones. The opposite is true. Prosen et al. 9] showed that a chaotic system is much more fidèle than a regular one, and suggested to use chaotic systems in quantum computing to suppress chaos.

It will be shown here that this is only part of the truth, and that for chaotic systems there is even a partial recovery of the fidelity at the Heisenberg time. This work extends the results by Gorin et al. [8] to the regime of strong perturbations using supersymmetry techniques. It is stressed that our results are generic and not restricted to random matrix systems. In a spin-chain model, e. g., the fidelity recovery has been observed recently as well [10].

Using the Brownian-motion model for the eigenvalues of random matrices introduced by Dyson many years ago 11], it will be shown that this behaviour has its direct analogue in the Debye-Waller factor of solid state physics. We shall sketch the calculation for the GUE only, and will just cite the result for the GOE. More details will be presented in a forthcoming paper 12 .

Let us start with an unperturbed Hamiltonian $H_{0}$, which is turned by a small perturbation into

$$
H_{\phi}=H_{0} \cos \phi+H_{1} \sin \phi
$$

It is assumed that both $H_{0}$ and $H_{1}$ have a mean level spacing of one, i. e. the variance of the off-diagonal elements is given by $N / \pi^{2}[13$. This particular choice of the perturbation guarantees that the mean density of states does not change with $\epsilon$. It will be come clear below that $\phi$ has to be of $\mathcal{O}(1 / \sqrt{N})$ to allow a well-defined limit $N \rightarrow \infty$. We therefore introduce $\epsilon=4 N \phi^{2}$ as the perturbation parameter. It follows for the fidelity amplitude

$$
f_{\epsilon}(\tau)=\left\langle\psi(0)\left|e^{2 \pi \mathrm{i}\left(c H_{0}+s H_{1}\right) \tau} e^{-2 \pi \mathrm{i} H_{0} \tau}\right| \psi(0)\right\rangle,
$$

where $\psi(0)$ is the wave function at the beginning, and $c=\cos \phi, s=\sin \phi$. The squared modulus of $f_{\epsilon}(\tau)$ yields the fidelity $F_{\epsilon}(\tau)$, originally introduced by Peres [1]. The calculation of the average of $F_{\epsilon}(\tau)$, however, is technically more involved and therefore not considered here.

For the present work the paper of Gorin et al [8] is of particular relevance. The author considered a slightly different parameter dependence

$$
H_{\lambda}=H_{0}+\lambda V
$$


where the variance of the off-diagonal elements of $V$ was assumed to be one. Comparison with equation (11) shows that the respective perturbation parameters are related via $\lambda=\sqrt{\epsilon} /(2 \pi)$.

In the paper by Gorin et al. 8] the Gaussian average of the fidelity amplitude was calculated in the regime of small perturbation strengths, correct up to $\mathcal{O}(\epsilon)$,

$$
\left\langle f_{\epsilon}(\tau)\right\rangle \sim e^{-\epsilon C(\tau)} .
$$

where $C(\tau)$ is given by

$$
C(\tau)=\frac{\tau^{2}}{\beta}+\frac{\tau}{2}-\int_{0}^{\tau} \int_{0}^{t} b_{2}\left(t^{\prime}\right) \mathrm{d} t^{\prime} \mathrm{d} t
$$

and $b_{2}(\tau)$ is the spectral form factor. $\beta$ is Dyson's universality index, i.e. $\beta=1$ for the Gaussian orthogonal ensemble (GOE), $\beta=2$ for the Gaussian unitary ensemble (GUE), and $\beta=4$ for the Gaussian symplectic ensemble (GSE). Equation (4) describes correctly the change from Gaussian to exponential decay with increasing perturbation strength. Equation (4) was the motivation for the introduction of $\epsilon$ as the perturbation parameter.

In chaotic systems the average of the fidelity amplitude over the initial state $\psi(0)$ reduces to a trace,

$$
\left\langle f_{\epsilon}(\tau)\right\rangle=\frac{1}{N}\left\langle\operatorname{Tr}\left[e^{2 \pi \mathrm{i}\left(c H_{0}+s H_{1}\right) \tau} e^{-2 \pi \mathrm{i} H_{0} \tau}\right]\right\rangle .
$$

$\left\langle f_{\epsilon}(\tau)\right\rangle$ may be written as a Fourier transform,

$$
\left\langle f_{\epsilon}(\tau)\right\rangle=\int d E_{1} d E_{2} e^{2 \pi \mathrm{i}\left(E_{1}-E_{2}\right) \tau} R_{\epsilon}\left(E_{1}, E_{2}\right)
$$

where

$$
\begin{aligned}
& R_{\epsilon}\left(E_{1}, E_{2}\right) \sim \\
& \quad \frac{1}{N}\left\langle\operatorname{Tr}\left(\frac{1}{E_{1-}-c H_{0}-s H_{1}} \frac{1}{E_{2+}-H_{0}}\right)\right\rangle,
\end{aligned}
$$

with $E_{ \pm}=E \pm$ i $\eta$. Using standard supersymmetry techniques [14], this can be written as

$$
\begin{aligned}
& R_{\epsilon}\left(E_{1}, E_{2}\right) \sim \\
& \quad \frac{1}{N} \int d[x] d[y] \sum_{n, m}\left(x_{n}^{*} x_{m}-\xi_{n}^{*} \xi_{m}\right)\left(y_{m}^{*} y_{n}-\eta_{m}^{*} \eta_{n}\right) \\
& \quad \times e^{-\mathrm{i}\left[\mathbf{x}^{\dagger} E_{1} \mathbf{x}-\mathbf{y}^{\dagger} E_{2} \mathbf{y}\right]} \\
& \quad \times\left\langle e^{\mathrm{i}\left[c \mathbf{x}^{\dagger} H_{0} \mathbf{x}-\mathbf{y}^{\dagger} H_{0} \mathbf{y}\right]}\right\rangle\left\langle e^{\mathrm{i} s \mathbf{x}^{\dagger} H_{1} \mathbf{x}}\right\rangle
\end{aligned}
$$

where $\mathbf{x}=\left(x_{1}, \xi_{1} \ldots x_{N}, \xi_{N}\right)^{T}, \mathbf{y}=\left(y_{1}, \eta_{1} \ldots y_{N}, \eta_{N}\right)^{T}$, and

$$
d[x]=\prod_{n} d x_{n} d x_{n}^{*} d \xi_{n} d \xi_{n}^{*}, \quad d[y]=\prod_{n} d y_{n} d y_{n}^{*} d \eta_{n} d \eta_{n}^{*}
$$

We adopt the usual convention using latin letters for commuting, and greek ones for anticommuting variables, respectively. Now the Gaussian average over the matrix elements of $H_{0}$ and $H_{1}$ can be performed elementary.

The subsequent steps (Hubbard-Stratonovich transformation, integration over the $x, y$ variables, saddle point integration etc.) are essentially the same ones as for the calculation of the spectral form factor (see e.g. chapter 10 of reference 15] for the GUE case). Details can be found in Ref. 12. We then arrive at the result

$$
\begin{aligned}
R_{\epsilon}\left(E_{1}, E_{2}\right) \sim & \frac{\rho^{2}}{N} \int_{0}^{\infty} d x \int_{0}^{1} d y \frac{x+y+x^{2}-y^{2}}{(x+y)^{2}} \\
& \times e^{-2 \pi \mathrm{i} \rho E(x+y)} e^{-\frac{\epsilon}{2} \rho^{2}(x+y)(1+x-y)}
\end{aligned}
$$

where $\rho=\sqrt{1-[\pi \bar{E} /(2 N)]^{2}}$ is the mean density of states, and $\bar{E}=\left(E_{1}+E_{2}\right) / 2, E=E_{1}-E_{2}$. Inserting this result into equation (7), and introducing $\bar{E}$ and $E$ as new integration variables, we get, fixing the constant of proportionality by the condition $f_{\epsilon}(0)=1$,

$$
\begin{aligned}
\left\langle f_{\epsilon}(\tau)\right\rangle= & \frac{1}{N} \int d \bar{E} \rho^{2} \int_{0}^{\infty} d x \int_{0}^{1} d y \frac{1+x-y}{x+y} \\
& \times \delta[\tau-\rho(x+y)] e^{-\frac{\epsilon}{2} \rho^{2}(x+y)(1+x-y)} .
\end{aligned}
$$

The $\bar{E}$ integration is nothing but an energy average. In the limit $N \rightarrow \infty$ only the band centre contributes where $\rho$ takes the constant value one. We may then discard this average and obtain

$$
\left\langle f_{\epsilon}(\tau)\right\rangle=\frac{1}{\tau} \int_{0}^{\operatorname{Min}(\tau, 1)} d y(1+\tau-2 y) e^{-\frac{\epsilon}{2} \tau(1+\tau-2 y)} .
$$

The integral is easily performed with the result

$$
\left\langle f_{\epsilon}(\tau)\right\rangle=\left\{\begin{array}{ll}
e^{-\frac{1}{2} \epsilon \tau}\left[s\left(\frac{1}{2} \epsilon \tau^{2}\right)-\tau s^{\prime}\left(\frac{1}{2} \epsilon \tau^{2}\right)\right], & \tau \leq 1 \\
e^{-\frac{1}{2} \epsilon \tau^{2}}\left[s\left(\frac{1}{2} \epsilon \tau\right)-\frac{1}{\tau} s^{\prime}\left(\frac{1}{2} \epsilon \tau\right)\right], & \tau>1
\end{array},\right.
$$

where

$$
s(x)=\frac{\sinh (x)}{x} .
$$

We have thus obtained an analytic expression for the GUE average of the fidelity amplitude for arbitrary perturbation strengths. In the limit of small perturbations it is in complete accordance with the result obtained by Gorin et al. 8].

The calculation for the GOE is done in exactly the same way. It is technically much more involved, but fortunately most of the work for this case has already been done by Verbaarschot, Weidenmüller, and Zirnbauer in their disseminating work [14]. In this way we get for the GOE average of the fidelity amplitude

$$
\left\langle f_{\epsilon}(\tau)\right\rangle=2 \int_{\operatorname{Max}(0, \tau-1)}^{\tau} d u \int_{0}^{u} \frac{v d v}{\sqrt{\left[u^{2}-v^{2}\right]\left[(u+1)^{2}-v^{2}\right]}}
$$




$$
\begin{aligned}
& \times \frac{(\tau-u)(1-\tau+u)}{\left(v^{2}-\tau^{2}\right)^{2}} \\
& \times\left[(2 u+1) \tau-\tau^{2}+v^{2}\right] e^{-\frac{1}{2} \epsilon\left[(2 u+1) \tau-\tau^{2}+v^{2}\right]} .
\end{aligned}
$$

To affirm the analytical findings, random matrix simulations were performed for all Gaussian ensembles including the symplectic one, which has not been treated analytically. The average was taken over up to 8000 random matrices of rank $N=500$ for $H_{0}$, and for each of them over 50 random matrices for $H_{1}$.

The results are shown in Figure 11 For the GOE and the GUE the numerical simulations are in perfect agreement with the analytical results for all $\epsilon$ values shown. For comparison, the fidelity amplitudes in the linear response approximation (4) are presented as well. For small perturbation strengths or small values of $\tau$ the linear response result is a good approximation, but the limits of its validity are also clearly illustrated.

For small perturbation strengths $\epsilon$ the decay of the fidelity is predominantly Gaussian which changes into a behaviour showing a cross-over from an exponential to a Gaussian decay at $\epsilon \approx 1$, in accordance with literature. The most conspicuous result of the present letter, however, is the partial recovery of the fidelity at the Heisenberg time $\tau=1$ which has not been reported previously to the best of our knowledge. This recovery is most pronounced for the GSE and least for the GOE. This is illustrated in Figure 2 showing a direct comparison of the fidelity amplitudes of the Gaussian ensembles for $\epsilon=30$. Preliminary simulations showed that the fidelity recovery is observed not only in the fidelity amplitude, but in the fidelity as well.

What is the origin of the surprising recovery? We believe that there is a simple intuitive explanation in terms of Dyson's Brownian motion model [1]. Since the mean density of states is kept constant during the parameter change, the eigenvalues of $H_{\phi}$ may be written as $E_{k}^{(\phi)}=k+\delta_{k}^{(\phi)}$, where $\delta_{k}^{(\phi)}$ fluctuates about zero. For strong perturbations the eigenvectors of the perturbed and unperturbed system are uncorrelated, and we get

$$
\left\langle f_{\epsilon}(\tau)\right\rangle=\frac{1}{N} \sum_{k l}\left\langle\left|R_{l k}\right|^{2}\right\rangle e^{2 \pi \mathrm{i} \tau(k-l)} W,
$$

where the $R_{k l}$ are obtained elementary from the eigenvectors, and $W$ is given by

$$
W=\left\langle e^{2 \pi \mathrm{i} \tau\left(\delta_{k}^{(\phi)}-\delta_{l}^{(0)}\right)}\right\rangle \approx e^{-(2 \pi \tau)^{2}\left\langle\delta^{2}\right\rangle} .
$$

In the second step a Gaussian approximation was applied. Within the framework of the Brownian motion model $\left\langle\delta^{2}\right\rangle$ is interpreted as the mean squared displacement of an eigenvalue from its equilibrium position. It is proportional to "temperature" $T$, which is just the reciprocal universality factor $\beta$, whence follows

$$
W=e^{-\alpha \tau^{2} T}
$$
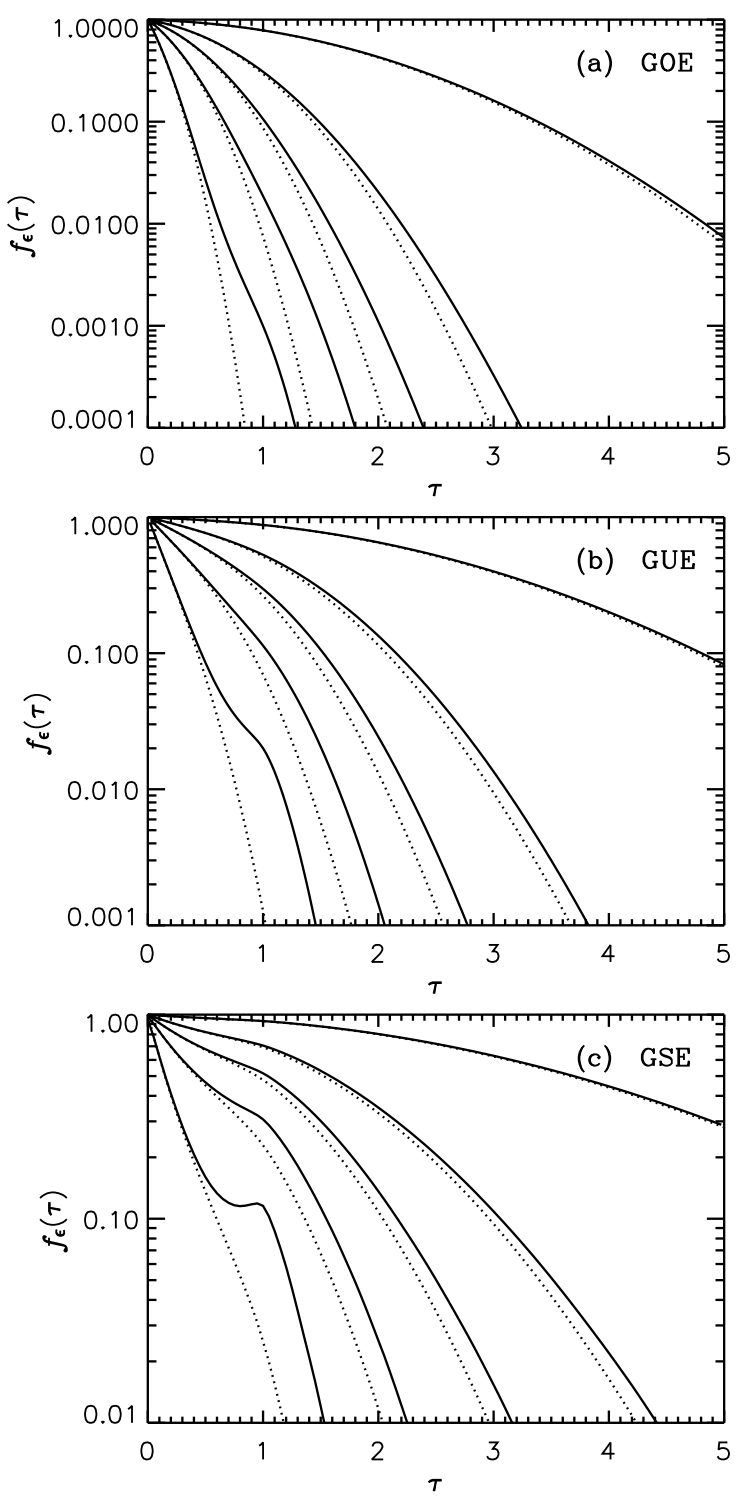

FIG. 1: Average of the fidelity amplitude $\left\langle f_{\epsilon}(\tau)\right\rangle$ for the GOE (a), the GUE (b), and the GSE (c) for $\epsilon=0.2,1,2,4$ and 10. $\tau$ is given in units of the Heisenberg time. For the GOE and the GUE the solid lines show the results of the analytical calculation, for the GSE of the numerical simulation. The dotted lines correspond to the linear response approximation (4). The numerical results are in agreement with the analytical results within the limits of the line strength.

with some constant $\alpha$. It follows from equation (17) that there is a revival of the fidelity at the Heisenberg time $\tau=$ 1 decreasing with "temperature" proportional to $e^{-\alpha T}$. This is exactly the behaviour illustrated in Figure 2

There is a perfect analogy to the temperature dependence of X-ray and neutron diffraction patterns in solid state physics. Caused by lattice vibrations the intensities of the diffraction maxima decrease with temperature 


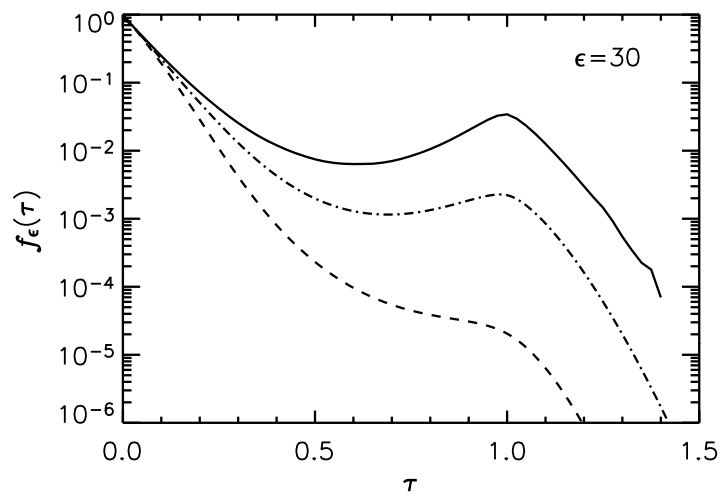

FIG. 2: Average of the fidelity amplitude $\left\langle f_{\epsilon}(\tau)\right\rangle$ for $\epsilon=$ 30. The dashed and dashed-dotted lines show the analytical results for the GOE and the GUE, respectively. The solid line corresponds to the numerical simulations for the GSE, reliable up to $10^{-3}$.

with a dependence described by the Debye-Waller factor

$$
W_{\mathrm{DW}}=e^{-\beta g^{2} T},
$$

where $\beta$ is another constant, and $g$ is the modulus of the reciprocal lattice vector characterizing the reflex (see e. g. appendix A of reference [16]). This is our justification to call $W$ a spectral Debye-Waller factor.

One may argue that due to equation (17) there should be revivals for all integer multiples of the Heisenberg time, which are not observed. This can be understood by considering the analogy between the spectral form factor

$$
K(\tau)=\frac{1}{N} \sum_{n, m} e^{2 \pi \mathrm{i}\left(E_{n}-E_{m}\right) \tau}
$$

and the structure factor in condensed matter,

$$
S(\vec{k})=\frac{1}{N} \sum_{n, m} e^{2 \pi \mathrm{i} \vec{k} \cdot\left(\vec{R}_{n}-\vec{R}_{m}\right)}
$$

where the $\vec{R}_{n}$ are the positions of the atoms, and $\vec{k}$ is a point in the reciprocal lattice. In liquids and glasses these peaks are smeared out, and the structure factor depends only on $k=|\vec{k}| . S(k)$ starts at zero for $k=0$, climbs up to two to three at the $k$ value corresponding to the reciprocal atomic distance, oscillates about one with decreasing amplitude for larger $k$ values, and approaches one in the limit $k \rightarrow \infty$ [17]. There is a striking similarity with the spectral form factor $K(\tau)$ of the GSE, the eigenvalue "crystal" with the lowest available temperature. $K(\tau)$, too, starts at zero for $\tau=0$, has a logarithmic singularity at the Heisenberg time $\tau=1$, and approaches one for $\tau \rightarrow \infty$. The oscillations found in $S(k)$, however, are absent in $K(\tau)$. Fidelity amplitude and spectral form factor thus do not show any structure at multiple integers of the Heisenberg time, in contrast to the corresponding condensed-matter quantities. The explanation is straightforward: Pauli's principle prevents the atoms from approaching too closely, whereas in the eigenvalue "crystal" such a lower limit does not exit.

If it is true that the universal spectral properties for all chaotic systems are described correctly by random matrix theory [18], and there is overwhelming evidence for this fact, then the exact expressions for the fidelity amplitude decay derived in this paper are universal and hold for all chaotic systems. But this means that the fidelity decay in this regime reflects the phase space properties of the system, but not its stability against perturbations as originally claimed by Peres [1]. Only in the regime of the Loschmidt echo the system stability shows up.

This paper has profited a lot from numerous discussions on the subject of fidelity with Thomas Seligman, Cuernavaca, Mexico, Thomas Gorin, Freiburg, Germany and Tomaž Prosen, Ljubljana, Slovenia. Thomas Guhr, Lund, Sweden is thanked for discussions of the supersymmetry aspects of this paper. The work was supported by the Deutsche Forschungsgemeinschaft.

[1] A. Peres, Phys. Rev. A 30, 1610 (1984).

[2] A. Abragam, The Principles of Nuclear Magnetism (University Press, Oxford, 1961).

[3] K. M. Frahm, R. Fleckinger, and D. L. Shepelyansky, Eur. Phys. J. D 29, 139 (2004).

[4] N. R. Cerruti and S. Tomsovic, Phys. Rev. Lett. 88, 054103 (2002).

[5] P. Jacquod, P. G. Silvestrov, and C. W. J. Beenakker, Phys. Rev. E 64, 055203 (2001).

[6] R. A. Jalabert and H. M. Pastawski, Phys. Rev. Lett. 86, 2490 (2001).

[7] H. M. Pastawski, P. R. Levstein, and G. Usaj, Phys. Rev. Lett. 75, 4310 (1995).

[8] T. Gorin, T. Prosen, and T. H. Seligman, New J. of Physics 6, 20 (2004).

[9] T. Prosen and M. Znidarič, J. Phys. A 35, 1455 (2002).

[10] C. Pineda, R. Schäfer, T. Prosen, and T. H. Seligman, to be published.

[11] F. J. Dyson, J. Math. Phys. 3, 1191 (1962).

[12] H.-J. Stöckmann and R. Schäfer, New J. of Physics 6, 199 (2004).

[13] M. L. Mehta, Random Matrices. 2nd edition (Academic Press, San Diego, 1991).

[14] J. J. M. Verbaarschot, H. A. Weidenmüller, and M. R. Zirnbauer, Phys. Rep. 129, 367 (1985).

[15] F. Haake, Quantum Signatures of Chaos. 2nd edition (Springer, Berlin, 2001).

[16] C. Kittel, Introduction to solid state physics (Wiley, New York, 1996).

[17] J. M. Ziman, Models of Disorder (University Press, Cambridge, 1979).

[18] O. Bohigas, M. J. Giannoni, and C. Schmit, Phys. Rev. Lett. 52, 1 (1984). 